\title{
Comparison of multispectral indexes extracted from hyperspectral images for the assessment of fruit ripening
}

\author{
L. Lleó ，J.M. Roger , A. Herrero-Langreo , B. Diezma-Iglesias , P. Barreiro \\ Departamento de Ciencia y Tecnologías Aplicadas a la Ingeniería Técnica Agrícola, E.U.I.T.A., 28040 Madrid, Spain \\ CEMAGREF, 361 rue Jean-François Breton BP 5095, 34196 Montpellier Cedex 5, France \\ Physical Properties and Advanced Techniques in Agrofood, LPF-TAG Rural Engineering Dept., Universidad Politécnica de Madrid, c/Ciudad Universitaria s/n, 28040 Madrid, Spain
}

Keywords:

Hyperspectral imaging

Vision

Ripeness

Non-destructive assessment

Quality

Peach

\begin{abstract}
A B S T R A C T
The present research is focused on the application of artificial vision to assess the ripening of red skinned soft-flesh peach ('Richlady'). Artificial vision allows a spatially detailed determination of the ripening stage of the fruit. The considered optical indexes ( Ind $_{1}$ and Ind $_{2}$, proposed in the present research, and Ind $_{3}$ and $\mathrm{I}_{\mathrm{AD}}$, proposed by other authors) are based on the combination of wavelengths close to the chlorophyll absorption peak at $680 \mathrm{~nm}$. Ind ${ }_{1}$ corresponds approximately to the depth of the absorption peak, and $\mathrm{Ind}_{2}$ corresponds to the relative absorption peak. An artificial image of each index was obtained by computing the corresponding reflectance images, which were acquired with a hyperspectral camera. All indexes were able to correct convexity (except for the just-harvested peaches and for $\operatorname{Ind}_{1}$ ). $\operatorname{Ind}_{2}$ is the preferred index; it showed the highest discriminating power between ripening stages and no influence of convexity. Ind $_{2}$ also allowed the differentiation of ripening regions within the fruits, and it showed the evolution of those regions during ripening.
\end{abstract}

\section{Introduction}

The quality assessment of fruits, particularly of peaches, is important for establishing the optimal harvest date, the best postharvest treatment, the cold storage temperature and the delay before consumption. Quality properties change rapidly from an unripe (low quality) to a ripe (high quality) stage. If the fruits are harvested too early, they will not ripen properly, and their quality at consumption will be poor (Crisosto, 1996; Moras, 1995).

The degree of ripeness can be assessed by destructive measurements (firmness measured by penetromy, Magness Taylor firmness, soluble solids content and acidity of the juice) and by non-destructive measurements. Non-destructive measurements may be carried out by examining optical properties (Lu and Peng, 2006; Ziosi et al., 2008), firmness estimated by impact (De Ketelaere et al., 2006; Diezma-Iglesias et al., 2004; Valero et al., 2007; Zude et al., 2006) or acoustic response (De Belie et al., 2000; Gómez et al., 2006).

The reference measure for firmness most commonly accepted by growers, packing houses, markets, and others is the Magness-Taylor penetration force (MT). However, it has been shown that this measurement lacks precision and has high instrumental and sampling variability. Firmness at harvest is one of the critical factors for peach management. At the packing station, softer fruits (below $20 \mathrm{~N}$ of MT) must be rejected due to their high susceptibility to damage, even if they are best for eating. At the same time, exceptionally hard fruits (over $50 \mathrm{~N}$ ) usually do not reach a minimum level of maturity and, therefore, cannot ripen properly. There is great interest, then, in classifying the fruits according to their firmness in a non-destructive way so that more homogeneous and resistant fruits can be chosen for the market.

Destructive and non-destructive measurements present parallel trends. However, non-destructive measurements are faster and can be implemented online. For peaches, the external colour and firmness advance in a similar or parallel pattern when monitored from the time of harvest to full consumer ripeness (Crisosto et al., 1995; Kader and Mitchell, 1989; Rood, 1957). In addition, peaches do not ripen in a homogeneous way. Depending on the cultivar, different areas of the fruit (e.g., the tip and shoulders) soften faster than others, and, therefore, these areas are more susceptible to damage and disease (Crisosto et al., 2001). Consequently, there is a need to develop non-destructive measuring techniques such as vision, which can provide a spatially detailed measurement of the degree of firmness and ripeness. The detection of the ripest areas through machine vision techniques could be employed as a rejection criterion in on-line classification equipment. Therefore machine vision could aid the classification of fruits according to their optimal des- 
tination: ripe samples to be sent to local markets, those which can be sent to distant markets, or unripe samples needing ripening treatments. In addition, machine vision can help to reduce the mistakes in manual classification.

The present research is focused on the application of vision to ripening assessment. Pigment changes during maturation and ripening can be detected through vision and reflectance techniques. Vision allows a spatially detailed determination of the ripening stage compared to spectrometry, which gives an average determination of an area of the fruit. The ripening process and external defects of fruits can be determined using multispectral and hyperspectral imaging (Leemans et al., 2002; Lu and Peng, 2006; Qin and Lu, 2008), providing better sensitivity than RGB imaging (Aleixos et al., 2002; Kleynen et al., 2003).

Hyperspectral vision is a powerful tool for searching the best combination of wavelengths regarding ripening. It could be applied in laboratory in an experimental framework to test and compare different wavelengths combinations. Once the best combination is chosen, it can be implemented in a multispectral vision camera.

In this paper we propose the comparison procedure for selecting the best combination. The present paper proposes two new indexes of ripening based on three wavelengths close to the chlorophyll absorption peak. The first part reviews spectral indexes regarding maturity and ripeness, and introduces these two new indexes from spectral considerations. The second part reports the materials and methods used for comparing these indexes with other existing indexes. The last part is devoted to the presentation and discussion of the results.

\section{Multispectral indexes}

\subsection{State of the art}

When assessing ripening with vision, it is crucial to identify the spectral changes associated with pigment evolution during ripening. Spectral indexes may consist of a single reflectance wavelength, differences or ratios between wavelengths or derivatives, or indices related to the red edge (a sharp change in reflectance between 680 and 750 related to chlorophyll content) (Xue and Yang, 2009). The use of ratios or differences is generally preferred because reflectance at one determined wavelength can be significantly affected by variable irradiance, background and geometric effects, sensors or other circumstances (Xue and Yang, 2009). Several indices have been developed for precision agriculture (Scotford and Miller, 2005). The normalised difference vegetation index NDVI is the most used index for the remote estimation of chlorophyll content. For ripeness, several studies have considered the chlorophyll band (Peng and Lu, 2007; Tu et al., 1995) or the water absorbance band (McGlone et al., 1997) to be the most relevant for maturity and firmness. Three areas in the visible range have been identified as the most important: $680 \mathrm{~nm}$ for chlorophyll, 450 and $480 \mathrm{~nm}$ for carotenoids and $535 \mathrm{~nm}$ for anthocyanin (Qin and Lu, 2008). Other spectrum areas, such as $500-550 \mathrm{~nm}$ for xanthophylls, have also been considered (Strack and Wray, 1989 cited by Merzlyak et al., 2003). Merzlyak et al. (2003) discuss the interactions of spectral features for different proportions of pigment content present in red and green fruits.

Ruiz-Altisent et al. (2006) showed that the reflectance associated with chlorophyll content (i.e., $680 \mathrm{~nm}$ ) is inversely related to firmness for several yellow- and red-skinned peach cultivars, including 'Richlady'. Ferrer et al. (2005) also observed significant differences in the reflectance spectra of Calanda peaches (clingstone, pale-yellowish, and non-melting fleshed cultivars) at $680 \mathrm{~nm}$. Reflectance at $680 \mathrm{~nm}$ for chlorophyll content presents the highest correlation with firmness in peaches (Lu and Peng, 2006). In fact, backscattered light at $677 \mathrm{~nm}$, characterised by four parameters extracted from their corresponding Lorentzian profile, is related to chlorophyll content, and it gives the highest correlation to flesh firmness in peach.

More recently, Qin and Lu (2008) explored the feasibility of using backscattered light in several fruits and vegetables to determine scattering and absorbance coefficients by employing hyperspectral images. The spectra of absorption coefficients were determined by the main pigments (chlorophyll, carotenoids and anthocyanin). The shape of the backscattered hyperspectral images presented a narrowing effect or a bottleneck at $675 \mathrm{~nm}$ in apple, pear, peach, kiwifruit, and green and pink tomatoes. This effect was due to the chlorophyll light absorption. This phenomenon did not occur with red tomatoes, which were the ripest.

Lleó et al. (2009) studied the behaviour of peach images acquired at $680 \mathrm{~nm}$ that covered the whole surface of the fruit. As the fruit ripens, the reflection in this band increases due to chlorophyll degradation. As a result, the histogram shifts towards brighter levels.

Several authors have studied different spectral indexes related to fruit ripening. Zude (2003) compared nine spectral indexes related to apple ripeness, including NDVI and ratios such as $698 \mathrm{~nm} / 780 \mathrm{~nm}$. Both obtained good results for certain cultivars. Qin and $\mathrm{Lu}$ (2008) presented a ratio of the absorption coefficient at $675 \mathrm{~nm}$ (chlorophyll) to that at $535 \mathrm{~nm}$ (anthocyanin) for the classification of tomato ripeness.

With peach, Ziosi et al. (2008) defined an optical index. This was an index of absorbance difference at two wavelengths, 670 and $720 \mathrm{~nm}\left(\mathrm{I}_{\mathrm{AD}}=\mathrm{A670-A720}\right)$. These wavelengths allowed them to differentiate between two maturity classes of 'Fayette' (yellow skin) peaches, which evolve differently during ripening and present different transcriptional profiles.

Another index, such as the reflectance ratio $670 \mathrm{~nm} / 800 \mathrm{~nm}$, have been shown to be useful for determining the ripeness of yellow clingstone peaches (Delwiche et al., 1987). Reflectances at $680 \mathrm{~nm}$ and at $800 \mathrm{~nm}$ have been also used by Sims and Gamon (2002) in remote sensing. The ratio of reflectance collected at 800 and $670 \mathrm{~nm}$ has been used to determine leaf chlorophyll content. Both wavelengths are related to the NDVI index because they are in the middle of the broad bands typically used in NDVI. Classification algorithms have been proposed based on red $(675 \mathrm{~nm})$ divided by infra-red $(800 \mathrm{~nm})$ images based on non-supervised clustering (Ward, 1963) whilst gathering the whole variability of ripeness in the harvest and post-harvest chain (Lleó et al., 2009). This classification presented a parallel evolution with firmness. As the fruits presented a higher classification score, they also showed lower firmness.

\subsection{Spectral considerations}

Fig. 1 presents the typical shapes of ripe and unripe peach reflectance spectra. The main difference is the chlorophyll absorption hole (around $680 \mathrm{~nm}$ ), which disappears as the fruit ripens. It is thus natural, as the above state of the art has shown, to use this peak. Nevertheless, the apparent reflectance is affected by two phenomena:

- The first is due to the variations of light scattering, which induce variations in the mean light path length. This produces a multiplicative variation of the observed reflectance.

- The second is due to some kind of specular reflection; that is, a variable part of the light does not enter the fruit and is reflected directly by the skin. This phenomenon produces an additive variation of the observed reflectance. 


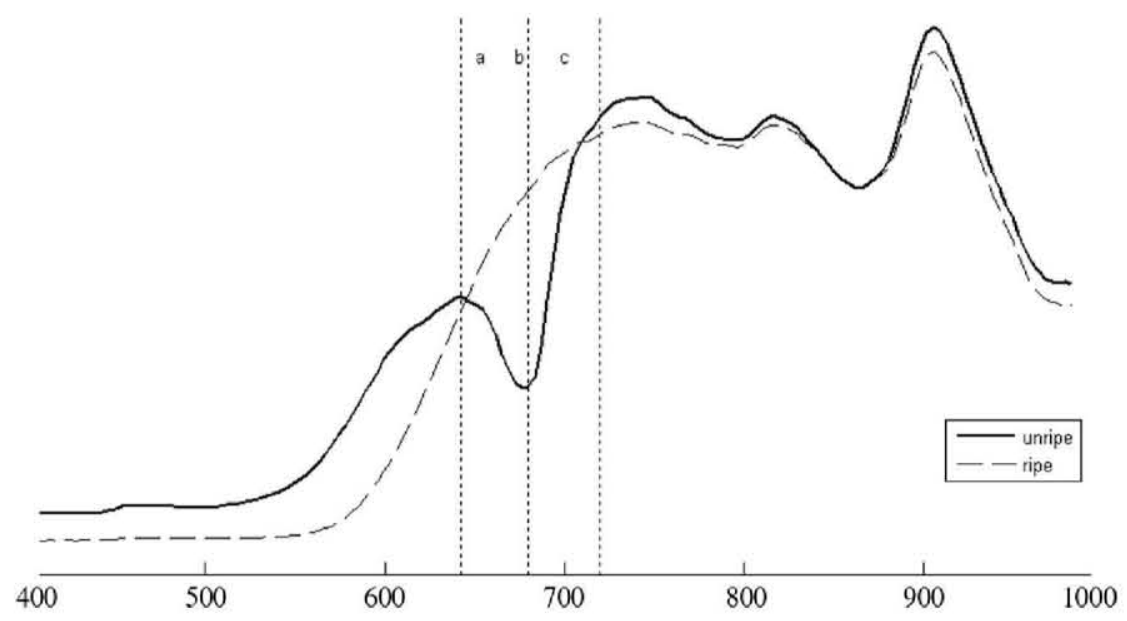

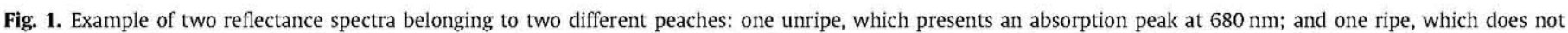
present any peak at $680 \mathrm{~nm}$. The $X$ axis corresponds to the wavelengths (nm). The $Y$ axis corresponds to the reflectance level of the spectra (arbitrary units).

As a first approximation, it is commonly considered that the multiplicative effect does not depend on the wavelength, whilst the additive effect is a function of the wavelength. These considerations yield the following expression of the apparent reflectance:

$R(\lambda)=K_{1} R_{0}(\lambda)+F(\lambda)$

where $\lambda$ is the wavelength, $R_{0}(\lambda)$ is the true (unknown) reflectance, $K_{1}$ is a constant and $F$ is a function (usually linear).

Next, the retrieval of the true reflectance from the measured signal involves two corrections that are respectively related to the multiplicative and additive effects:

- A normalisation that consists of dividing the spectrum by a quantity that is affected by the multiplicative effect but is not linked to the chemical composition of the sample. For example, the Standard Normal Variate (SNV) process (Barnes et al., 1993) performs a normalisation by the standard deviation of the spectrum.

- A baseline subtraction that consists of removing the background trend from each spectrum. This operation can be achieved explicitly, for example, by identifying the baseline (linear, quadratic, etc.) or by performing a $k$-order differentiation. The latter solution is commonly performed by means of the Savitsky and Golay algorithm (Gorry, 1990), which performs smoothing simultaneously with differentiation. The second order of differentiation is usually preferred because it removes the linear baselines and magnifies the peaks.

The application of such corrections to the chlorophyll peak with a limited number of wavelengths can be achieved on the basis of three reflectances: $R_{\mathrm{a}}, R_{\mathrm{c}}$ and $R_{\mathrm{b}}$. These reflectances correspond respectively to the sides and the bottom of the peak, as illustrated in Fig. 1. Two levels of correction can be applied, yielding two indexes:

- The baseline correction should be carried out by an approximation of the second derivative on the peak, as performed by Ind $_{1}$ :

$$
\operatorname{Ind}_{1}=R_{a}+R_{c}-2 R_{b}
$$

- The correction for the multiplicative effect is done in $\mathrm{Ind}_{2}$ by dividing $I_{1} d_{1}$ by the mean value of the two reflectances $R_{\mathrm{a}}$ and $R_{\mathrm{C}}$ (which should not depend on the ripening):

$\operatorname{Ind}_{2}=\left(R_{a}+R_{c}-2 R_{b}\right) /\left(\left(R_{a}+R_{c}\right) / 2\right)$

After the simplification (dividing the numerator and denominator by $R_{\mathrm{a}}+R_{\mathrm{c}}$ ) and the removal of the constant terms and factors, Ind $_{2}$ becomes:

$$
\operatorname{Ind}_{2}=R_{b} /\left(R_{a}+R_{c}\right)
$$

\subsection{Proposed indexes}

On the basis of the above considerations, the present research proposes to compute the new indexes, $\operatorname{Ind}_{1}$ and $\operatorname{Ind}_{2}$, on the following wavelengths: $\lambda_{\mathrm{b}}=680 \mathrm{~nm}, \lambda_{\mathrm{a}}=640 \mathrm{~nm}$ and $\lambda_{\mathrm{c}}=730 \mathrm{~nm}$. Ind $_{1}$ corresponds approximately to the depth of the absorption peak at $680 \mathrm{~nm}$, and $\mathrm{Ind}_{2}$ corresponds to the relative absorption peak. Ind corrects for the additive effect, and $\operatorname{Ind}_{2}$ corrects for the additive plus multiplicative effects, as explained in the previous paragraph.

\section{Materials and methods}

\subsection{Materials}

Red-skinned, soft-flesh 'Richlady' peaches were harvested from an orchard in the south of France. Five commercially mature fruits were selected by expert assessment based on apparent colour and firmness (Fig. 2).

Hyperspectral images of the selected fruits were acquired before and after the following ripening process: refrigerated storage for four days at $10^{\circ} \mathrm{C}$ and ripening for three days at room temper-

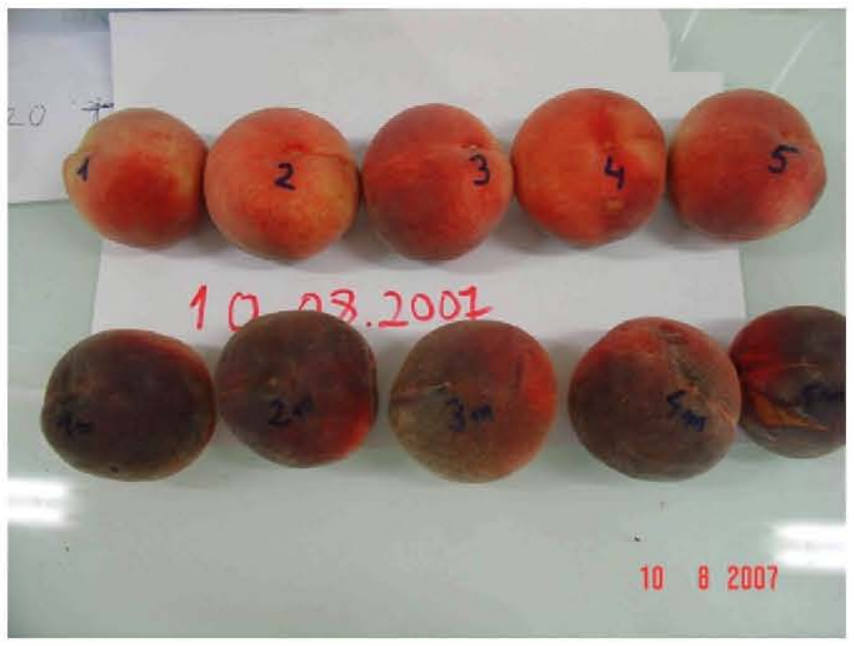

Fig. 2. 'Richlady' peaches with different maturity stages. Fruits $1-5$, first line, correspond to unripe samples; marked fruits $1-5 \mathrm{~m}$, second line, correspond to ripe samples. 
ature $\left(20^{\circ} \mathrm{C}\right)$. Two images were acquired for each fruit, corresponding to each of the two sides delimited by the suture of the fruit. One of the images was not correctly acquired, so that, 9 fruit sides have been finally analysed. Each of these 9 samples was analysed before and after the ripening process.

\subsection{Hyperspectral measurements}

Hyperspectral images were acquired from each side of the fruit. The vision system was provided by a HYSPEX VNIR 1600-pixel push-broom camera (Hyspex, Norsk Elektro Optikk AS, Norway). The light was provided by a classical tungsten lamp. An image of a white panel provided a blank reference, which permitted the reflectance spectra of each pixel to be calculated.

\subsection{Images computed based on chlorophyll indexes}

On the basis of the description in Section 2, the following four indexes were computed on the whole image of the fruit sides:
(a) Ind $_{1}=\left(R_{730}+R_{640}\right)-2 * R_{680}$
(b) Ind $_{2}=R_{680} /\left(R_{640}+R_{730}\right)$
(c) Ind $_{3}=R_{675} / R_{800}$
(d) $\mathrm{I}_{\mathrm{AD}}=\log 10\left(R_{720} / R_{670}\right)$

Ind ${ }_{1}$ and $\operatorname{Ind}_{2}$ are the new indexes, and the other two were employed before; Ind $_{3}$ was proposed by Sims and Gamon (2002) and Lleó et al. (2009), and $I_{A D}$ was proposed by Ziosi et al. (2008). An artificial image of each index was obtained computing the corresponding reflectance images. The region of interest (the fruit) was previously segmented from the background using the Otsu method (Otsu, 1979).

\subsection{Comparison criteria}

Two analyses were applied for comparing the indexes:

(a) Ripening sensing. The objective of this analysis was to determine which indexes better discriminate the ripening process, for each fruit. As the number of pixels is not the same for each fruit, Wilks' lambda $A$ (Wilks, 1960) has been preferred to $F$ (Fisher) statistics. Wilk's lambda is a criterion that can be used for comparison purpose. It was computed for each pair of fruits before and after ripening as the ratio between the inter-group variance and the residual variance. Nine pairs of fruits were considered, and thus, nine values of $A$ were obtained per index. This statistic measures the individual discriminative power of a variable. For each variable $(Y)$ Wilk's lambda $(\Lambda)$ was computed as the ratio of the sum of squares between groups $\left(\mathrm{SS}_{\mathrm{B}}\right)$ and the sum of squares within groups $\left(\mathrm{SS}_{\mathrm{W}}\right)$.

$A=\frac{\mathrm{SS}_{\mathrm{B}}}{\mathrm{SS}_{\mathrm{W}}}$

In turn, the sum of squares within groups $\left(S S_{W}\right)$ was computed as:

$\mathrm{SS}_{\mathrm{W}}=\sum_{j=1}^{q} \sum_{i=1}^{n_{j}}\left(Y_{i j}-\bar{Y}_{j}\right)^{2}=\sum_{i=1}^{n_{1}}\left(Y_{i 1}-\bar{Y}_{1}\right)^{2}+\sum_{i=1}^{n_{2}}\left(Y_{i 2}-\bar{Y}_{2}\right)^{2}$

adding up the sum of squares within group (j): group 1 (pixels of a fruit before ripening) and group 2 (pixels of the same fruit after ripening), being $q$ the number of groups ( $q=2) ; n_{j}$ the corresponding number of pixels, $n_{1}$ and $\mathrm{n}_{2} ; \bar{Y}_{j}$, the mean for each group $j$ and $Y_{i j}$, the value of each pixel (i) from group $(j)$ for the variable $Y$. Since, each time there was compared two groups, there were considered two means of groups $\bar{Y}_{1}, \bar{Y}_{2}$.

The sum of squares between groups $\left(\mathrm{SS}_{\mathrm{B}}\right)$ was computed as:
$\mathrm{SS}_{\mathrm{B}}=\sum_{j=1}^{q}\left(\bar{Y}_{j}-\bar{Y}_{\mathrm{TOTAL}}\right)^{2} n_{j}=n_{1}\left(\bar{Y}_{1}-\bar{Y}_{\mathrm{TOTAL}}\right)+n_{2}\left(\bar{Y}_{2}-\bar{Y}_{\text {TOTAL }}\right)$

Where $\bar{Y}_{\text {TOTAL }}$ is the mean of the merged population gathering both groups.

$A$ ranges from 0 to $\infty$. The higher the $A$, the more discriminative is the variable $Y$.

(b) Robustness of the indexes in relation to convexity of the fruit. The objective of this comparison was to analyse which indexes are affected by the convexity. A normalised artificial image (not shown) for each index was computed by subtracting to every pixel of the image the index value at the geometrical centre of the fruit. Next, the correlation coefficient $R$ was computed between the index of each pixel and its distance to the geometrical centre. $R$ was computed for each index and each ripening stage, considering the nine fruit images pooled together.

\subsection{Statistical discrimination tests}

In order to test and confirm the discrimination ability of the indexes, all pixels from every peach were pulled together before (non-ripened group) and after ripening (ripened group). ANOVA was carried out for each index comparing these two groups. In addition, a very previous attempt of classification of the spectra was carried out. For that, a calibration set ( 5000 spectra from ripened group, and 5000 from non-ripened group) was randomly selected from the images. The centroids of the two groups, nonripened and ripened, were computed for each index. Then it was calculated Euclidean distances of each pixel of the fruit images to both centroids. After that each pixel was assigned to the nearest class according to those distances. The observed and previous assignments of all the pixels were compared with the classification based on the Euclidean distance. This comparison was made for each index.

All analyses were carried out using Matlab $^{\mathbb{Q}}$ (version 7.0; Math Works, Inc., USA).

\section{Results}

\subsection{Objective criteria for comparison}

\subsubsection{Ripening sensing. Wilks' lambda computation}

Fig. 3 shows the box plots of the nine values ( $y$ axis) for the four indexes ( $x$ axis). Each box corresponds to the inter-quartile range, and the median is indicated by a horizontal line. The lines extend from each end of the boxes to show the whole range. Considering the median, the global performance of the four indexes is as follows: $A\left(\operatorname{Ind}_{2}\right)>A(\operatorname{Ind} 1)>A\left(\mathrm{I}_{\mathrm{AD}}\right)>\Lambda\left(\mathrm{Ind}_{3}\right)$. Considering the interquartile interval, it appears that $\Lambda\left(\operatorname{Ind}_{1}\right)$ and $\Lambda\left(\mathrm{Ind}_{3}\right)$ vary to a much lesser degree than $\Lambda\left(\mathrm{I}_{\mathrm{AD}}\right)$ and $\Lambda\left(\mathrm{Ind}_{2}\right)$. The same indexes order is found in Table 1 according to $F$ of Fisher in ANOVA test. In addition, it is also shown that all indexes are able to discriminate between the two ripening stages.

Table 2 shows $A$ for each pair of fruits and each index. The highest $A$ values always correspond to $\operatorname{Ind}_{2}$ (values ranging from 1 to 10.6). Ind $_{1}$ ( $A$ values from 0.7 to 3.1 ), $\mathrm{I}_{\mathrm{AD}}\left(0.08\right.$ to2.7), and $\mathrm{Ind}_{3}$ $\left(0.03\right.$ to 0.98 ) differed greatly from Ind $_{2}$. The $A$ ranges overlapped for these three indexes.

Fig. 4 represents the average spectra of the representative fruits of high $\Lambda$ (fruits 1 and 6 ), which probably have a higher ripening evolution, and low $\boldsymbol{A}$ (fruits 3 and 5), which probably have a low ripening evolution. It is to note that the behaviour of the spectra of some of the areas of the image might differ from the average spectra of the whole image of the fruit. Consequently, whilst $A$ takes into account all the spectra of all the pixels, and therefore variations within fruits, the average spectrum of each fruit can only 


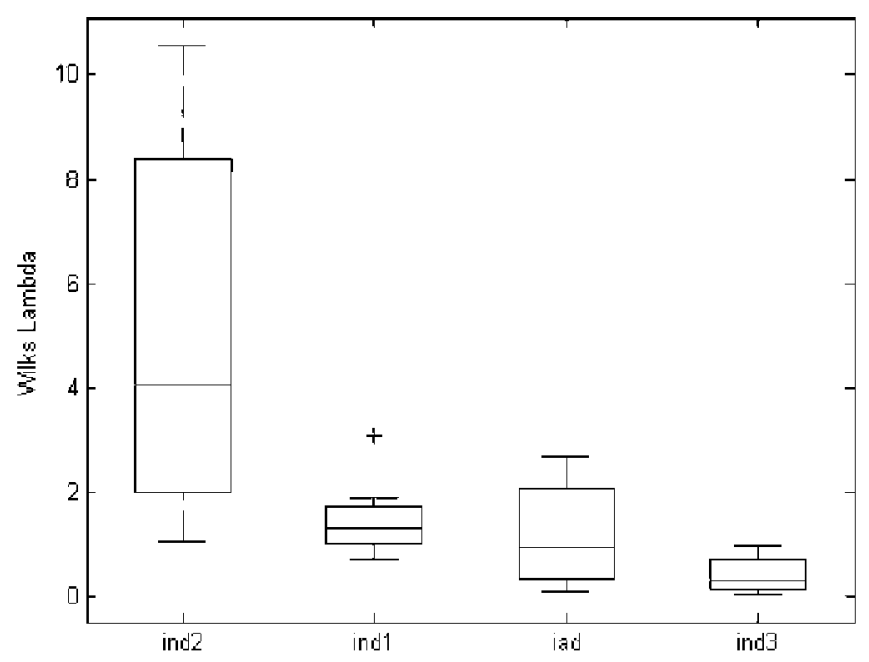

Fig. 3. Wilks' lambda $A$ inter-quartile ranges for the nine fruits before and after ripening ( $y$ axis) for the four indexes ( $x$ axis). Median values are indicated by horizontal lines in the boxes.

\section{Table 1}

Comparison of ANOVA results for the artificial images of each index. All pixels of the fruit images were pooled together for the analysis, categorised in two groups: one group before $n=40,071$ and other group after ripening $n=34,957$.

\begin{tabular}{lllll}
\hline & $\operatorname{Ind}_{1}$ & $\operatorname{Ind}_{2}$ & $\operatorname{Ind}_{3}$ & $\mathrm{I}_{\mathrm{AD}}$ \\
\hline $\begin{array}{l}F \text { (Fisher) } \\
p \text { level }\end{array}$ & 168,620 & 256,960 & 43,616 & 90,031 \\
& 0 & 0 & 0 & 0 \\
\hline
\end{tabular}

Table 2

Wilks' lambda value for each fruit and index before and after ripening. Bold case indicates the lowest $\boldsymbol{A}$, whereas cursive case indicates the highest $\boldsymbol{A}$.

\begin{tabular}{llrllll}
\hline Fruit & $\operatorname{lnd}_{1}$ & $\operatorname{lnd}_{2}$ & $\operatorname{lnd}_{3}$ & $\mathbf{I}_{A D}$ & $\begin{array}{l}\text { Number of } \\
\text { pixels before rip. }\end{array}$ & $\begin{array}{l}\text { Number of } \\
\text { pixels after rip. }\end{array}$ \\
\hline 1 & 3.09 & 9.59 & 0.68 & 1.48 & 3855 & 3490 \\
2 & 1.41 & 4.61 & 0.84 & 2.68 & 3967 & 3531 \\
3 & $\mathbf{1 . 3 1}$ & 2.32 & $\mathbf{0 . 0 4}$ & $\mathbf{0 . 0 8}$ & 4306 & 3840 \\
4 & 1.90 & 3.59 & 0.15 & 0.38 & 4301 & 3790 \\
5 & 1.15 & 4.05 & $\mathbf{0 . 0 3}$ & $\mathbf{0 . 2 2}$ & 4892 & 4207 \\
6 & 1.07 & 10.56 & 0.54 & 1.86 & 5057 & 4219 \\
7 & 0.78 & 1.08 & 0.19 & 0.56 & 4839 & 4217 \\
8 & $\mathbf{0 . 7 2}$ & $\mathbf{1 . 0 4}$ & 0.31 & 0.95 & 4780 & 4066 \\
9 & $\mathbf{1 . 6 9}$ & 7.98 & 0.98 & 2.62 & 4074 & 3597 \\
\hline
\end{tabular}

be used to compare single examples of ripe and unripe spectra. As an example, fruit 6 presents high variation of the relative absorption peak at $680 \mathrm{~nm}$, that is Ind $_{2}$, and it also presents high evolution rate (Table $2, A=10.56$ ). This effect (Fig. 4) is not so clear for fruit 1 maybe due to the above mentioned fact that we are representing only the average spectrum. On the other hand, fruits 3 and 5 present not so high change of $\operatorname{Ind}_{2}$ (lower values of $A$, than fruits 6 and 1 ) and graphically they present also low variation of the relative absorption peak considering the average spectra before and after ripening.

\subsubsection{Robustness of the indexes in relation to convexity of the fruit}

Table 3 shows the correlation coefficient between the radial positions and the index values for all pixels of all the fruits before and after ripening. When only the single $680 \mathrm{~nm}$ wavelength is used, the correlation is very high. This clearly demonstrates the interest of the normalised indexes for increasing the robustness of the measurement. Amongst the indexes, only Ind $_{1}$ seems to be affected by the radial position for unripe fruits, as confirmed by the images in Fig. 5(1), where concentric circles appear.

\subsection{Description of the computed images}

Index images before and after ripening for the same fruits are shown in the left column of Fig. 5. The variability within the fruit is studied through the average and standard deviation per fruit, plotted in the right column of Fig. 5.

\subsubsection{Ind $_{1}$}

Ind $_{1}$ is based on the non-normalised second derivative. This index decreased with maturity as expected (the absorption peak at $680 \mathrm{~nm}$ disappears with ripening). It also showed a certain convexity effect, which disappeared after ripening. In just-harvested group the coefficient of correlation between the intensity value and the distance to the geometric centre of the fruit was $R=-0.64$, whilst in after ripening group it was $R=-0.12$.

The variability within the fruits is lower in the ripened fruits when compared to the fruits at harvest (Fig. 5(1)). The vertical blue and red lines show a parallel evolution (the original maturity of the fruits could determine fruit ripening at the end of the process).

\subsection{2. $\operatorname{lnd}_{2}$}

This index presented the lowest variability within the fruits. The blue and red lines were completely parallel. Some ripened regions (on the top and near the shoulders) were allocated to the same areas before and after ripening. The intensity values and area of those regions increased after ripening, as can be observed in the third, fourth, seventh and eighth fruits from left to right on the top of the fruit (Fig. 5(2)).

\subsubsection{Ind}

This index increased with ripening. The average and range lines overlapped between the groups before and after ripening, whilst high variability was observed within the fruits. These images also show ripened regions with values that expanded and increased during the process (ripening areas on fruits 3, 4, 6 and 9 from left to right in Fig. 5(3)). These regions were generally located on the top of the fruits and did not exactly match the differentiated areas detected by $\operatorname{Ind}_{2}$.

\subsection{4. $I_{A D}$}

This index decreased along the ripening process. It showed a similar behaviour to $\mathrm{Ind}_{3}$. It had overlapping, differentiated ripening regions that evolved along the ripening process and a similar ranking of fruits according to ripening. The riper spots were usually located on the shoulders (Fig. 5(4)).

\section{Discussion}

All of the indexes reflected the ripening evolution. The average range of each fruit evolved as expected for all indexes (increasing for $I_{2} \mathrm{I}_{2}$ and $\mathrm{Ind}_{3}$ and decreasing for $\mathrm{Ind}_{1}$ and $\mathrm{I}_{\mathrm{AD}}$ ) along the ripening process. This behaviour can be explained by the common inclusion of reflectance at $680 \mathrm{~nm}$, the chlorophyll absorption peak, which increased with ripening due to the chlorophyll degradation. This behaviour has been observed by other studies using spectra acquired by a spectroradiometer (Ferrer et al., 2005) and backscattering profiles acquired in narrow bands of the skin in hyperspectral images (Qin and Lu, 2008; Lu and Peng, 2006). The above mentioned authors showed the relationship between ripeness and reflectance/absorbance at $680 \mathrm{~nm}$. Lu and Peng (2006), especially, showed that the backscattered light is related to firmness. None of these measurements were spatially detailed, whereas the present research shows imaging of the whole fruit. As reported by other authors (Crisosto et al., 2001), some differentiated regions of ripening could be detected on the images of multispectral-based 
(1) Ind $_{1}$

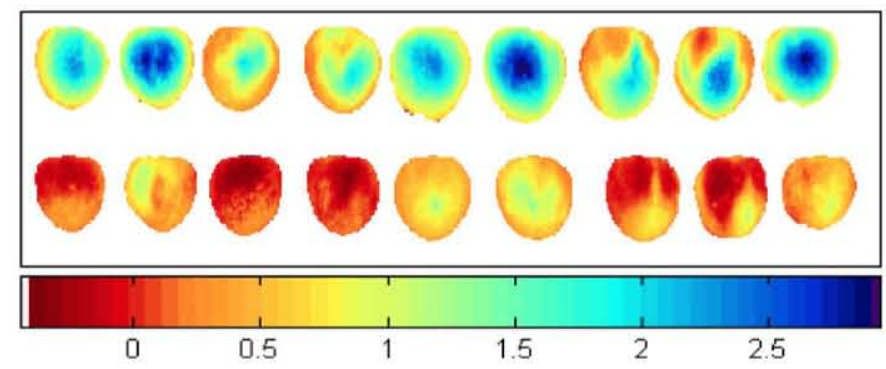

(2) $\operatorname{Ind}_{2}$

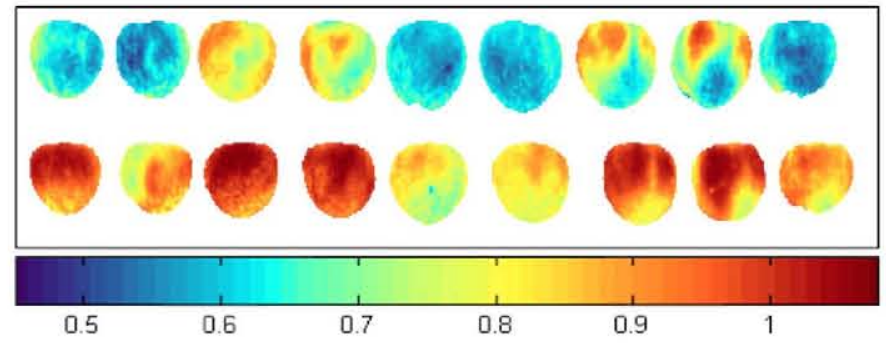

(3) $\operatorname{Ind}_{3}$

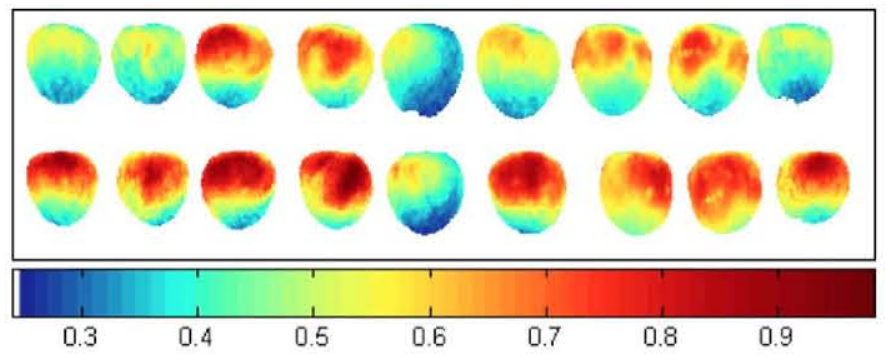

(4) $\mathrm{I}_{\mathrm{AD}}$
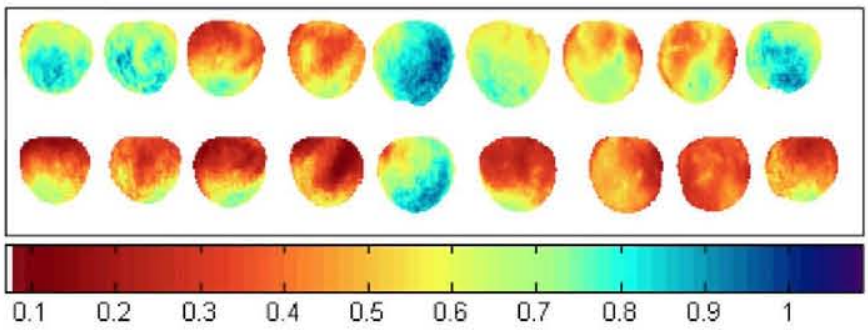
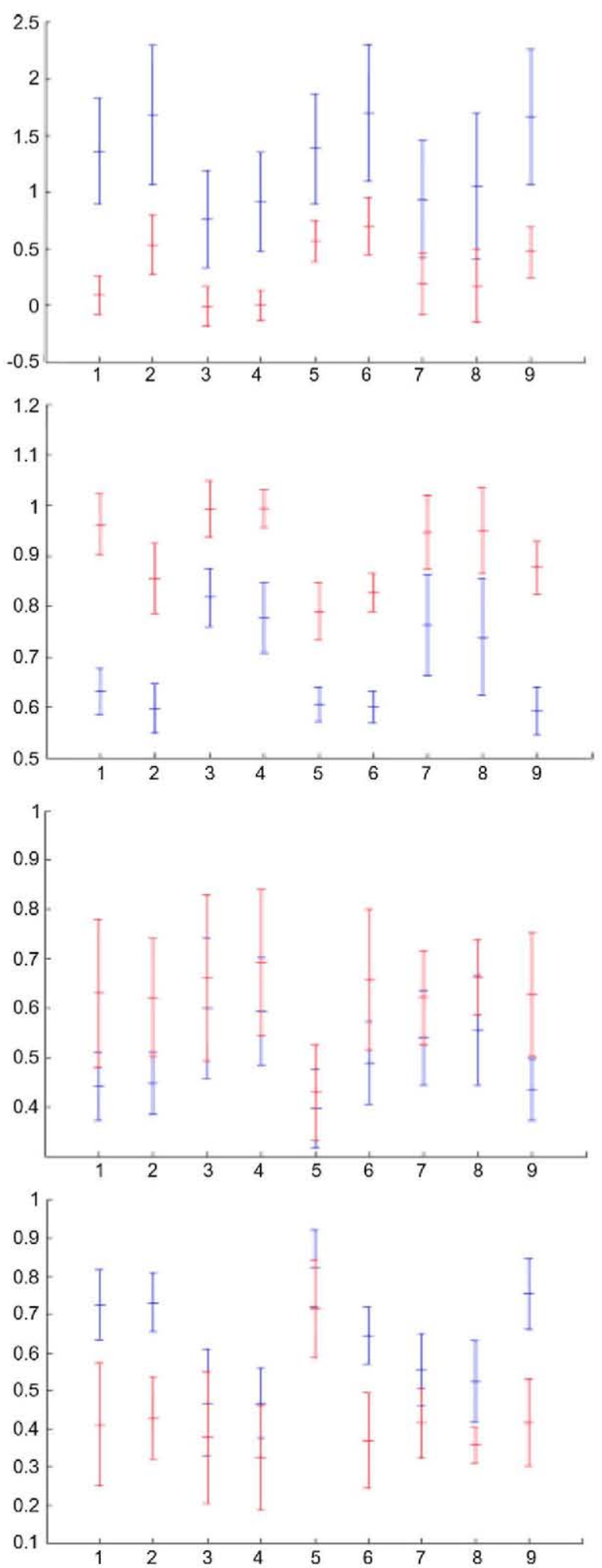

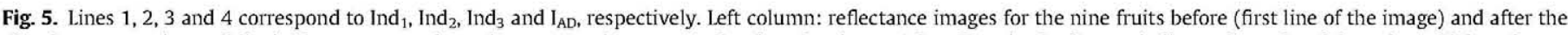

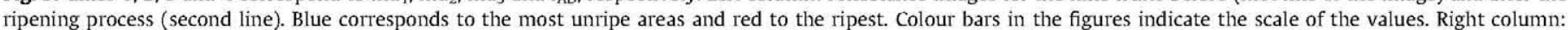

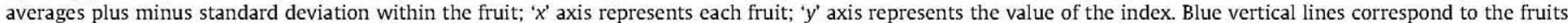

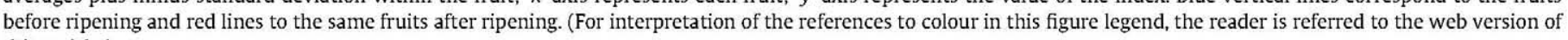
this article.)

effect which, as mentioned in the Section 2.2, can be associated with variations in the mean light path length. The other indexes are not sensitive for either ripened or non-ripened fruits. Furthermore, the angle between incident and reflected light changes according to the position of the pixel in relation to the centre of the fruit, because the centre of the fruit matches the optical centre of the image. Therefore, the path length of the light through the fruit changes. When the fruit is unripe, the curvature of the spectra 
at chlorophyll hole is significantly different than zero. Ind $_{1}$ ("the depth of the hole') is high and is sensitive to path length variations. The lack of sensitivity of Ind $_{1}$ to convexity for ripe fruits could be related to the effect of ripeness on the spectra. When the fruit is ripe, the curvature of the spectrum disappears; Ind $_{1}$ decreases and becomes less sensitive to the path length. The value tends to be constant and low and thus less sensitive to the radial position.

\subsection{Which index presented a higher discriminate power?}

The applied test Wilks lambda (Table 2) and $F$ of Fisher (Table 1) gave concordant results. In both cases Ind $_{2}$ was the most discriminating index: it presented the highest $F$ value and median of Wilk's lambda. Furthermore the classifications of all pixels ( $n=75,028$ ) based on the Euclidean distances to the centroids (non-ripened and ripened group), showed that Ind 2 presented the highest percentage of correctly classified (83\%). The rest of the indexes achieved the following percentages: $79 \% \operatorname{Ind}_{1}, 74 \% \mathrm{I}_{\mathrm{AD}}$ and $68 \%$ Ind $_{3}$.

Wilks' Lambda is supposed to be related to fruit evolution. A high lambda value supposes high variance before and after ripening compared to the variability within fruits and, therefore, will have a higher discriminating power between the ripening stages.

Ind $_{2}$ presented the highest median of lambda $(\Lambda=4)$, followed by $\operatorname{Ind}_{1}(\boldsymbol{A}=1.5), \mathrm{I}_{\mathrm{AD}}(\boldsymbol{\Lambda}=1)$, and $\operatorname{Ind}_{3}(\Lambda=0.5)$ (Table 2 and Fig. 3). Furthermore, Ind $_{2}$ always produced the highest $A$ in the nine pairs of fruits (Table 2 ) and the greatest separation between each pair of fruits along the fruits' evolution (Fig. 5, line 2). Thus, Ind $_{2}$ could be the best index regarding discrimination ability and $\mathrm{Ind}_{3}$ the worst (maximum overlapping between the fruits before and after ripening). Because $\operatorname{Ind}_{2}$ is normalised (and therefore more robust than $I_{1} d_{1}$ ) and focuses exactly on the shape of the spectrum around the chlorophyll zone, it characterises the ripening stage well. This result confirms the necessity of compensating for both multiplicative and additive effects when considering reflectance images.

\subsection{Why is there more variation in $A$ for some indexes than others?}

Let us divide the effect of ripening on spectra into a "mean effect", which affects all fruits similarly, and a "fruit-specific effect". It seems that Ind ${ }_{2}$ and $I_{A D}$ are sensitive to both effects, because (i) they present the highest differences between the median values of $A$, and (ii) their inter-quartile intervals are the largest. This higher sensitivity of $I_{2} d_{2}$ and $I_{A D}$ may be explained by their ability to detect spatial variations inside the fruits.

\subsection{Which index presented more variation in $A$ ?}

Fruits present different rates of evolution. A higher $A$ for a fruit suggests faster ripening under the chosen postharvest conditions.

Considering each index (each column in Table 2), the different fruits presented different $A$ probably because of their evolution rate. Thus, some fruits seemed to evolve faster (high $A$, fruits 1 and 6) than others (low $A$, fruits 3 and 5).

Ind $_{2}$ showed the highest range of $A$, and, therefore, it may be the best index for detecting the different evolution rates.

\subsection{Why are some fruits judged differently by certain indexes?}

Fruits are judged differently by the indexes. Focusing on the $A$ ranges and values of individual fruits, the indexes can be grouped into two pairs: Ind $_{1}$ and $I_{2} d_{2}$, and Ind $_{3}$ and $I_{A D}$ (Table 2 ). The lowest values of $A$ were obtained for the same fruit within each pair of indexes: fruit 8 for $\operatorname{Ind}_{1}$ and $\operatorname{Ind}_{2}$, and fruit 3 according to $\operatorname{Ind}_{3}$ and
$\mathrm{I}_{\mathrm{AD}}$. In addition, high $\Lambda$ values were computed for fruit 1 according to Ind $_{1}$ and Ind $_{2}$ and for fruits 2 and 9 according to Ind $_{3}$ and $\mathrm{I}_{\mathrm{AD}}$.

These different behaviours could be because the indexes characterised two kinds of changes in the spectra; that is, Ind $_{1}$ and Ind $_{2}$ focused on the shape of the chlorophyll absorption area, and Ind $_{3}$ and $I_{A D}$ focused on the difference or ratio between the red and infra-red zones. It is notable that the ripe spectra presented a global decrease, especially at the NIR (Fig. 4). This is surely due to a global change in the skin reflectance. Because $\mathrm{I}_{\mathrm{AD}}$ and $\mathrm{Ind}_{3}$ are based on the difference between the NIR (above $730 \mathrm{~nm}$ ) and the red zone (around $680 \mathrm{~nm}$ ), the decreasing NIR reflectance lowers $\mathrm{I}_{\mathrm{AD}}$ (and increases $\mathrm{Ind}_{3}$ ) artificially. Ind $\mathrm{I}_{3}$ and $\mathrm{I}_{\mathrm{AD}}$, then, mix the change at the chlorophyll zone with the decreasing NIR area, which can be influenced by other factors apart from ripening, like water loss.

Fruit 5, for example, was judged differently by both groups of indexes. Ind $_{3}$ and $\mathrm{I}_{\mathrm{AD}}$ did not detect the evolution (low $A, 0.03$ and 0.22 , respectively), whereas $\operatorname{Ind}_{2}$ did detect the evolution $(A=4.05)$. The decrease in the spectrum could have changed the ripening characterisation, and it could have influenced this disagreement between indexes (Fig. 4d). Furthermore, fruit 1 is judged to have fast evolution by $\operatorname{Ind}_{2}(A=10.6)$ and slow evolution by $\operatorname{Ind}_{3}(\Lambda=0.68)$ and $\mathrm{I}_{\mathrm{AD}}(\Lambda=1.48)$. Fruit 1 also presented a general decrease at the NIR region.

Fruits that showed quicker evolution (Fig. 4, first line, fruits 1 and 6) presented a greater change of shape at the chlorophyll absorption area. Fruits that showed less evolution (Fig. 4, second line, lower $A$, like fruits 3 and 5 ) presented a weak change. Fruit 6 , for example, is judged to have high evolution by $\operatorname{Ind}_{2}(A=9.5)$, some evolution by $\operatorname{Ind}_{1}(A=3.1)$ and low evolution by $\operatorname{Ind}_{3}$ $(A=0.54)$, whilst fruit 6 presents a high difference at the chlorophyll region.

\section{Conclusions}

The present research proposes two new indexes, $\operatorname{Ind}_{1}$ and $\mathrm{Ind}_{2}$, to be compared with two previously used indexes, $\operatorname{Ind}_{3}$ (Lleó et al., 2009; Sims and Gamon, 2002) and IAD (Ziosi et al., 2008). All of these indexes were extracted from hyperspectral images, and their corresponding multispectral images were computed. Hyperspectral image system is employed for searching the best combination of wavelengths regarding the problem of ripening sensing. Once the indexes are proposed, a much cheaper system, multispectral, could be employed to compute the respective artificial images, because only few (three or four) wavelengths are actually needed.

Great differences were found in their ability to discriminate ripening stages through Wilks' $A$. The Wilks' $A$ values were computed between the index values of each fruit before and after ripening. Ind $_{2}$ showed the highest $A$ for all the fruits because $\operatorname{Ind}_{2}$ is a normalised index and is focused on the shape at the chlorophyll absorption peak, at $680 \mathrm{~nm}$.

All indexes were able to correct the convexity effect (the correlation coefficient $R$ between the index values for all pixels and their radial positions was $0.01-0.48$ ), except for the just-harvested peaches and for $\operatorname{Ind}_{1}(R=-0.66)$. The $680 \mathrm{~nm}$ images, however, were greatly affected by convexity ( $R$ around 0.75 or 0.8 ), and the proposed indexes were able to correct this effect.

As a conclusion, Ind $_{2}$ is the preferred index. It showed a clear parallel evolution of average and range values, lower variability within the fruits, better discrimination between ripening stages and no convexity effect. In addition, Ind $_{2}$ allowed the differentiation of ripening regions within the fruits and showed the evolution of those regions during ripening.

Further analysis could be done by comparing reference measurements, such as firmness, from differentiated, ripened regions 
detected by the studied indexes vs. reference values from the remaining part of the fruit. To validate the obtained results, an extended analysis with an increased sample size would be necessary.

\section{Acknowledgements}

This work was carried out at the Universidad Politécnica de Madrid (Spain) within the TAGRALIA-CM program and the ISAFRUIT ("Increasing fruit consumption through a trans-disciplinary approach leading to high quality produce from environmentally safe, sustainable methods") - EU 6th FP IP project. The authors wish to thank Professor F. Riquelme from CEBAS-CESIC (Spain) for his help with the selection of samples.

\section{References}

Aleixos, N., Blasco, J., Navarrón, F., Moltó, E., 2002. Multispectral inspection of citrus in real-time using machine vision and digital signal processors. Computers and Electronics in Agriculture 33 (2), 121-137.

Barnes, R.J., Dhanod, M.S., Lister, S.J., 1993. Correction to the description of standard normal variate (snv) and de-trend transformations in practical spectroscopy with applications in food and beverage analysis - 2nd edition. Journal of Near Infrared Spectroscopy $1,185-186$.

Crisosto, C.H., Mitchell, F.G., Johnson, S., 1995. Factors in fresh market stone fruit quality. Post Harvest News and Information 6 (2), 17-21.

Crisosto, C.H., 1996. Optimum procedures for ripening stone fruit. Management of ripening fruit. Postharvest Horticulture $9,28-30$.

Crisosto, C.H., Slaughter, D.C., Garner, D., Boyd, J., 2001. Stone fruits critical bruising thresholds. Journal American Pomological Society 55 (2), 76-81.

De Belie, N., Schotte, S., Lammertyn, J., Nicolai, B., De Baerdemaeker, J., 2000. Phpostharvest technology: firmness changes of pear fruit before and after harvest with the acoustic impulse response technique. Journal of Agricultura Engineering Research 77 (2), 183-191.

De Ketelaere, B., Howarth, M.S., Crezee, L., Lammertyn, J., Viaene, K., Bulens, I., De Baerdemaeker, J., 2006. Postharvest firmness changes as measured by acoustic and low-mass impact devices: a comparison of techniques. Postharvest Biology and Technology 41 (3), 275-284.

Delwiche, M., Tang, S., Rumsey, J.W., 1987. Color and optical properties of clingstone peaches related to maturity. American Society of Agricultural Engineers 30 (6) 1873-1879.

Diezma-Iglesias, B., Ruiz-Altisent, M., Barreiro, P., 2004. Detection of internal quality in seedless watermelon by acoustic impulse response. Biosystems Engineering 88 (2), 221-230.

Gómez, A.H., He, Y., Pereira, A.G., 2006. Non-destructive measurement of acidity, soluble solids and firmness of satsuma mandarin using vis/NIR-spectroscopy techniques. Journal of Food Engineering 77 (2), 313-319.

Ferrer, A., Remón, S., Negueruela, A.I., Oria, R., 2005. Changes during the ripening of the very late season Spanish peach cultivar Calanda: feasibility of using CIELAB coordinates as maturity indices. Scientia Horticulturae 105 (4), 435-446.

Gorry, P.A. 1990. General least-squares smoothing and differentiation by the convolution (Savitzky-Golay) method. Analytical Chemistry $62,570-573$.

Kader, A.A., Mitchell, F.G., 1989. Postharvest physiology. In: La Rue, J.H., Johnson, R.S. (Eds.), Peaches, Plums and Nectarines: Growing and Handling for Fresh Market. Department of Agriculture and Natural Resources Publication, University of California, pp. 158-164

Kleynen, O., Leemans, V., Destain, M.F., 2003. Selection of the most efficient wavelength bands for 'jonagold' apple sorting. Postharvest Biology and Technology 30 (3), 221-232.
Leemans, V., Magein, H., Destain, M.F., 2002. Ae-automation and emerging technologies: on-line fruit grading according to their external quality using machine vision. Biosystems Engineering 83 (4), 397-404

Lu, R., Peng, Y., 2006. Hyperspectral scattering for assessing peach fruit firmness. Biosystems Engineering 93 (2), 161-171.

Lleó, L., Barreiro, P., Ruiz-Altisent, M., Hetrero, A., 2009. Multispectral images of peach related to firmness and maturity at harvest. Journal of Food Engineering 93 (2), 229-235.

McGlone, V.A., Abe, H., Kawano, S., 1997. Kiwifruit firmness by near infrared light scattering. Journal of NIR Spectroscopy 5, 83-89.

Merzlyak, M.N., Solovchenko, A.E., Gitelson, A.A., 2003. Reflectance spectral features and non-destructive estimation of chlorophyll, carotenoid and anthocyanin content in apple fruit. Postharvest Biology and Technology 27 (2), 197-211.

Moras, P., 1995. Peach elements d'un matrisse de la qualite apres recolte. Infoscentre technique interprofessionel des fruits et légumes (Ctifl) 112, 34-37.

Otsu, N., 1979. A threshold selection method from gray-level histograms. IEEE Transactions on Systems, Man \& Cybernetics 9, 62-66.

Peng, Y., Lu, R., 2007. Prediction of apple fruit firmness and soluble solids content using characteristics of multispectral scattering images. Journal of Food Engineering $82(2), 142-152$.

Qin, J., Lu, R., 2008. Measurement of the optical properties of fruits and vegetables using spatially: resolved hyperspectral diffuse reflectance imaging technique. Postharvest Biology and Technology 49, 355-365.

Rood, P., 1957. Development and evaluation of objective maturity indices for California freestone peaches. American Society of Agricultural and Biological Engineers 70, 104-112.

Ruiz-Altisent, M., Lleó, L., Riquelme, F., 2006. Instrumental quality assessment of peaches: fusion of optical and mechanical parameters. Journal of Food Engineering 74 (4), 490-499.

Scotford, I.M., Miller, P.C.H., 2005. Applications of spectral reflectance techniques in northern European cereal production: a review. Biosystems Engineering 90 (3), $235-250$.

Sims, D.A., Gamon, J.A., 2002. Relationships between leaf pigment content and spectral reflectance across a wide range of species, leaf structures and developmental stages. Remote Sensing of Environment 81 (2-3), 337-354.

Strack, D., Wray, V., 1989. Anthocyanins. In: Harborne, J.B., Dey, P.M. (Eds.), Methods in Plant Biochemistry. Academic Press, pp. 326-352.

Tu, K., De Busscher, R., De Baerdemaeker, J., Schrevens, E., 1995. Using laser beam as light source to study tomato and apple quality non-destructively. In: Proceeding of the Food Processing Automation IV Conference, Chicago, IL, pp. $528-536$.

Valero, C., Crisosto, C.H., Slaughter, D., 2007. Relationship between nondestructive firmness measurements and commercially important ripening fruit stages for peaches, nectarines and plums. Postharvest Biology and Technology 44 (3), $248-253$.

Ward Jr., J.H., 1963. Hierarchical grouping to optimize an objective function. Journal of the American Statistical Association 58 (301), 236-244.

Wilks, S., 1960. Multidimensional Scatter. Standford Press.

Xue, L., Yang, L., 2009. Deriving leaf chlorophyll content of green-leafy vegetables from hyperspectral reflectance. ISPRS Journal of Photogrammetry and Remote Sensing 64 (1), 97-106.

Ziosi, V., Noferini, M., Fiori, G., Tadiello, A., Trainotti, L., Casadoro, G., Costa, G., 2008. A new index based on vis spectroscopy to characterize the progression of ripening in peach fruit. Postharvest Biology and Technology 49 (3), 319-329.

Zude, M., 2003. Comparison of indices and multivariate models to non-destructively predict the fruit chlorophyll by means of visible spectrometry in apple fruit. Analytica Chimica Acta 481 (1), 119-126.

Zude, M., Herold, B., Roger, J.-M., Bellon-Maurel, V., Landahl, S., 2006. Nondestructive tests on the prediction of apple fruit flesh firmness and soluble solids content on tree and in shelf life. Journal of Food Engineering 77 (2), 254260. 